Check for updates

Cite this: Chem. Sci., 2020, 11, 596

๑ All publication charges for this article have been paid for by the Royal Society of Chemistry

Received 26th September 2019 Accepted 23rd November 2019

DOI: $10.1039 / c 9 s c 04859 f$

rsc.li/chemical-science

\section{Combining hydrophilic and hydrophobic environment sensitive dyes to detect a wide range of cellular polarity $\dagger$}

\author{
Sang Jun Park, \$Vinayak Juvekar, + Jae Hyung Jo and Hwan Myung Kim (D) *
}

Intracellular polarity is an important parameter of pathological and biological phenomena of cells; abnormal polarities are associated with diabetes, neurological diseases, and cancer. However, previously reported polarity probes have issues with quantitatively detecting intracellular polarities, can measure only a limited range of polarities, and can only detect specific intracellular regions. Here, we developed a novel two-dye system, RPS-1, that contains a new "turn-on" polarity probe (Dye1) based on a spiropyran intramolecular ring closing-opening system activated in polar protic solvents, and a benzothiadiazole containing dye (Dye3), which emits only in non-polar solvents with a large stoke shift. Individually, Dye1 and Dye3 selectively localized to lysosome and lipid droplets, respectively; however, combining these dyes, which have completely different characteristics, via a piperazine linker resulted in the staining of various intracellular organelles. Therefore, as Dye1 and Dye3 have the same absorption but different emissions, combining them resulted in a ratiometric polarity probe that could quantitatively measure a wider polarity range inside the cell using a single excitation source. In addition, ratiometric imaging using our RPS-1 probe to quantitatively detect the distribution of polarity in different cell lines indicated that lysosomes were the most polar organelles in the cell.

\section{Introduction}

In the intracellular environment, polarity, viscosity, temperature, redox status, and $\mathrm{pH}$ parameters are critical for the initiation and maintenance of the physical and chemical behaviors of biomolecules, because their distribution, spatial arrangement, and composition within the cell are heterogeneous. ${ }^{1,2}$ Intracellular polarity, in particular, is key to various cellular processes such as cell proliferation, immune system regulation, increases in the number of local membranes, stimulation of cell migration, and vectorial transport of molecules across the cell layer. ${ }^{3,4}$ Each organelle of the cell has an optimal polarity depending on its role and the polarity changes in real time as the intracellular environment changes..$^{5-7}$ The pathological activity of the cell changes with its polarity, and abnormal polarity is related to diabetes, neurological diseases, and cancer..$^{8-11}$ Therefore, detecting cellular polarity is important in research on pathological and biological phenomena.

The only way to observe intracellular polarity is to use optical imaging; therefore, various fluorescent probes have been developed to detect the polarity of microenvironments. ${ }^{12-27}$

Department of Chemistry, Department of Energy Systems Research, Ajou University, Suwon 443-749, Korea.E-mail: kimhm@ajou.ac.kr

$\dagger$ Electronic supplementary information (ESI) available: Synthesis, additional methods, and figures (Fig. S1-S32 and Tables S1-S3). See DOI: 10.1039/c9sc04859f \$ S. J. Park and V. Juvekar contributed equally to this work.
Most reported polarity probes are based on intramolecular charge transfer (ICT) and show shifts in emission wavelengths based on changes in the polarity of a cell's surroundings. However, most of these probes are solvatochromic and have disadvantages including the fact that the fluorescence efficiency decreases sharply as the solvent polarity increases, limiting the detection range in hydrophobic environments. In addition, the reported probes can only detect specific structures in a cell, such as the mitochondria, ${ }^{12,13}$ lysosomes, ${ }^{14-17}$ endoplasmic reticulum (ER), ${ }^{18-20}$ lipid droplets, ${ }^{21-23}$ and cell membrane. ${ }^{24-26}$ Therefore, these probes are limited to detecting the polarity of a particular organelle or a limited zone and have difficulties in imaging the distribution of polarity over the entire cell. Recently, a polarity sensitive probe for detecting lysosomes and lipid droplets was reported. ${ }^{27}$ Even though the probe showed good selectivity, its applications are limited because two different excitation wavelengths must be used to observe each organelle, making it difficult to show the polarity distribution in a cell in real time and thus to quantify the polarity of the organelles. To maintain and regulate appropriate cellular activities, multiple organelles exchange materials and transmit intracellular signals. ${ }^{28,29}$ Therefore, studying complex subcellular organelle interactions requires a highly sensitive polarity probe that labels more than two organelles and can quantitatively detect a wide range of cellular polarities.

Here, we introduce a ratiometric probe (RPS-1) that overcomes the limitations described above. We first synthesized D- 
(a)

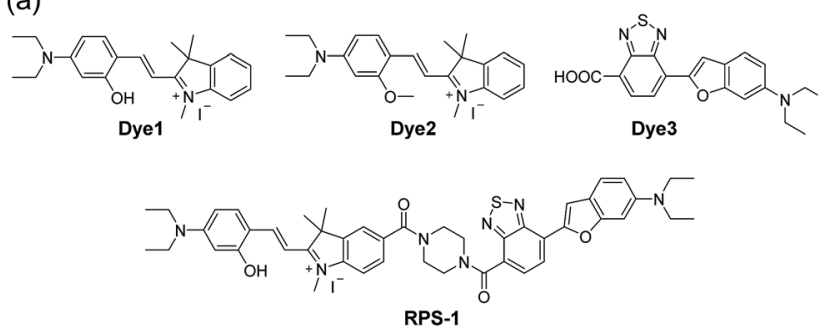

(b)

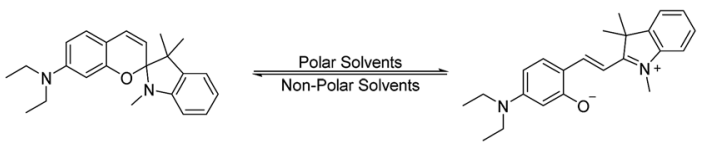

Scheme 1 (a) Chemical structures of Dye1, Dye2, Dye3, and RPS-1. (b) The proposed polarity sensing mechanism of Dye1.

$\pi$-A type dipolar compounds by treating aminosalicylaldehyde with indolium and a benzothiadiazole acceptor expecting to produce ICT-based dyes, denoted as Dye1 and Dye3 (Scheme 1). Dye3 was characterized as an ICT-based dye whose fluorescence largely shifted to the red-region, but decreased dramatically following an increase in solvent polarity (see below, Fig. S6†). Interestingly, Dye1 showed an "off-on" characteristic in hydrophobic and hydrophilic solvents as its structure underwent a ring opening/closing cycle. To cover a wide range of polarities, we formed a ratiometric probe that combined these two dyes, which were sensitive to hydrophilic and hydrophobic environments (Dye1 and Dye3), respectively. This probe had different emission windows and could be excited with a single wavelength source. The fluorescence intensity ratio of RPS-1 was dramatically changed between the yellow and red windows, depending on the polarity, and was highly correlated with the $E_{\mathrm{T}}^{\mathrm{N}}$ value, a parameter that indicates the polarity of a solvent. Therefore, because RPS-1 could quantitatively detect the polarity of various organelles in cells, the probe revealed that lysosomes were the most polar organelle in cells and that lipid droplets were the most non-polar.

\section{Results and discussion}

Polarity probes were synthesized by condensing salicylaldehyde and 1,2,3,3-tetramethyl-3 $H$-indolium iodide under ethanol (EtOH) conditions. The detailed synthesis method, yield, and NMR for each intermediate are described in the ESI. $\dagger$

The synthesized Dye1 was measured for absorbance and fluorescence intensity using solvents with various polarities ranging from toluene to water (Fig. 1). The charge transfer of Dye1 was blocked in the nonpolar solvent due to its closed-ring structure and it absorbed in the short wavelength region at $328 \mathrm{~nm}$. However, in solvents with a high polarity, such as those above EtOH, Dye1 opened the ring of the closed structure and increased the conjugation bridge, thus recovering the charge transfer from the diethylaniline to the indolium salt (Scheme 1b). As a result, the absorbance gradually decreased at $328 \mathrm{~nm}$ but increased at a long wavelength of $549 \mathrm{~nm}$. Similarly, there
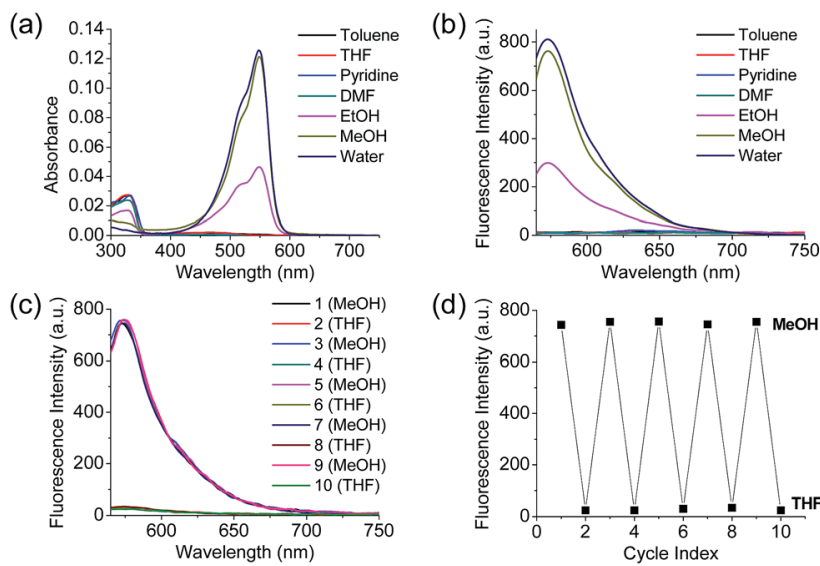

(d)

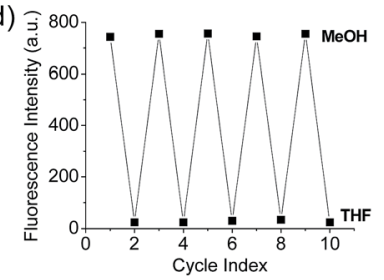

Fig. 1 (a) Absorption spectra and (b) fluorescence spectra of Dye1 in various polar and non-polar solvents. (c) Fluorescence spectrum and (d) fluorescence intensities of Dye1 with the polarity of $\mathrm{MeOH}$ and THF solvents reversed for five cycles. All concentrations for the spectral measurements were $1 \mu \mathrm{M}$, the excitation wavelength was $552 \mathrm{~nm}$, and the fluorescence intensity was acquired at $573 \mathrm{~nm}$.

was no fluorescence intensity for Dye1 at an excitation of $552 \mathrm{~nm}$ in the nonpolar solvent, but strong fluorescence did occur in the $573 \mathrm{~nm}$ region in the highly polar environment.

Dye2 was synthesized to block cyclization by introducing a methyl group into the hydroxyl group to confirm that the change in absorbance and fluorescence based on the solvent polarity of Dye1 was the result of the intramolecular cyclizationopening system. Dye2 showed strong absorbance at approximately $550 \mathrm{~nm}$ in all solvents, regardless of their polarity, and showed no absorbance at a wavelength of $328 \mathrm{~nm}$ (Fig. S1 $\dagger$ ). The fluorescence intensities of Dye 2 at the $552 \mathrm{~nm}$ excitation source were observed at $580 \mathrm{~nm}$ in all the solvents.

To confirm that the intramolecular cyclization and opening of Dye1 could be reversed by changing the solvent polarity, Dye1 was added to both $\mathrm{MeOH}$ and THF as the solvents five times and the fluorescence spectra were measured (Fig. 1c and d). A constant change in fluorescence was observed under each set of conditions tested; thus, it was confirmed that the intramolecular cyclization and opening for Dye1 were reversible with the change in solvent polarity.

The ${ }^{1} \mathrm{H}$ NMR spectra were analyzed in nonpolar and polar solvents to further demonstrate the cyclization-opening system of the polarity probes (Fig. 2). In the non-polar $\mathrm{CDCl}_{3}$ solvent, Dye1 was a closed structure and the intramolecular chiral center was present. Due to this, the two gem-dimethyl groups in the spirocyclic form, with $\delta 1.14$ and $1.32 \mathrm{ppm}$, were well separated. However, in the polar solvent environment of $\mathrm{CD}_{3} \mathrm{OD}: \mathrm{D}_{2} \mathrm{O}=$ $1: 1$, the spirocyclic form of Dye1 underwent an intramolecular structural change in the opened structure and the chiral center disappeared. The separated gem-dimethyl was observed as one peak at $\delta 1.72 \mathrm{ppm}$. At the same time, the amine of indoline became positively charged as the polarity of the solvent increased. As a result, the methyl of the amine was shifted from $\delta 2.74 \mathrm{ppm}$ to $\delta 3.71 \mathrm{ppm}$. In the medium polarity environment of $\mathrm{CDCl}_{3}: \mathrm{CD}_{3} \mathrm{OD}=1: 1$, the NMR data showed that both the 


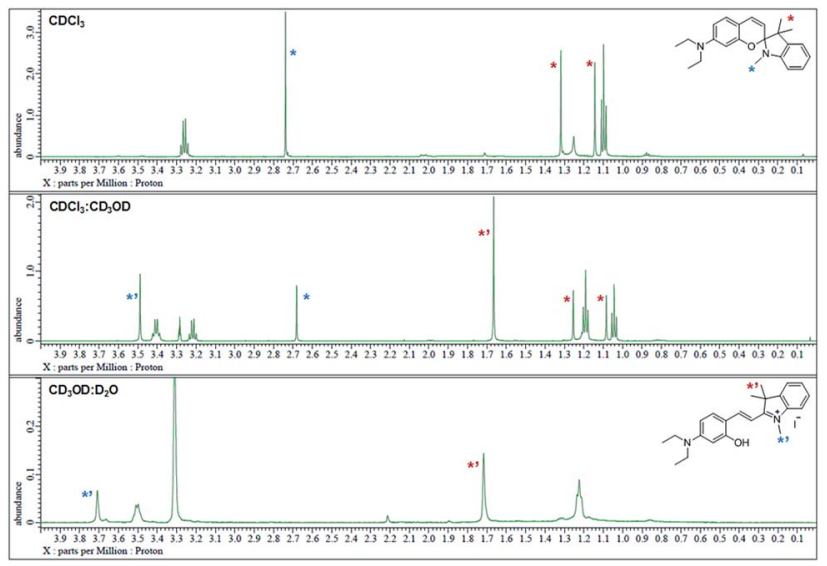

Fig. $2{ }^{1} \mathrm{H}$ NMR spectrum ( $\delta$ 0.0-4.0 region) of Dye1 in $\mathrm{CDCl}_{3}$, $\mathrm{CDCl}_{3}: C D_{3} \mathrm{OD}=1: 1(\mathrm{v} / \mathrm{v})$ and $\mathrm{CD}_{3} \mathrm{OD}: \mathrm{D}_{2} \mathrm{O}=1: 1(\mathrm{v} / \mathrm{v})$ solvents.

closed and opened structures coexist. A clear $\mathrm{C}=\mathrm{C}$ double bond cis proton was observed in the aromatic region; it was due to the spirocyclic ring in the nonpolar environment $(J=10.3 \mathrm{~Hz})$. In the polar environment, it was difficult to observe a clear trans proton but there was a clear and large downfield shift due to the strong electron withdrawing effect. The NMR peaks and chemical structures of Dye1 for each polarity environment were assigned (Fig. S2 †).

The absorbance and fluorescence spectra of the probes in the range of $\mathrm{pH} 4$ to $\mathrm{pH} 10$ were recorded to determine whether the polarity probe was affected by $\mathrm{pH}$ (Fig. S3 $\dagger$ ). The results showed that all polarity probes were always in the open form in aqueous solution, regardless of the change in $\mathrm{pH}$.

Each polarity probe was incubated in HeLa cells for $30 \mathrm{~min}$. Under the $552 \mathrm{~nm}$ excitation wavelength, Dye1 and Dye2 emitted bright fluorescence, but the intracellular locations of each probe were different. Dye1 tended to stain a specific vesicle in the cell, whereas Dye2, which was always in the open form, stained a wide area in the cell, which might be mitochondria (Fig. 3). As a result of the co-localization experiments with each of the polarity probes with LysoTracker Green, Dye1 significantly overlapped with a Pearson coefficient value of 0.94 , suggesting that the organelles where this probe was located were lysosomes (Fig. 3a). However, Dye2 had a low Pearson coefficient with LysoTracker (Fig. S5†), which was not overlapping, and instead showed a high overlap with MitoTracker Green (Fig. 3b). The mitochondrial inner membrane is known to have a proton concentration gradient for ATP synthesis and a negative charge of about $-180 \mathrm{mV} .^{30}$ As a result, small molecules with positive charge tend to accumulate inside the mitochondria due to electrostatic attraction. Therefore, Dye2, which was positively charged due to the opened structure, was located in the mitochondria for the same reason. However, it has been reported that cyclized indolines like Dye1 can be located in lysosomes in a tertiary amine form that can act as a proton receptor. ${ }^{31}$ These results suggested that either Dye1 preferred to be located in the lysosomes or the probe was distributed at various locations within the cell but only the lysosomes could be
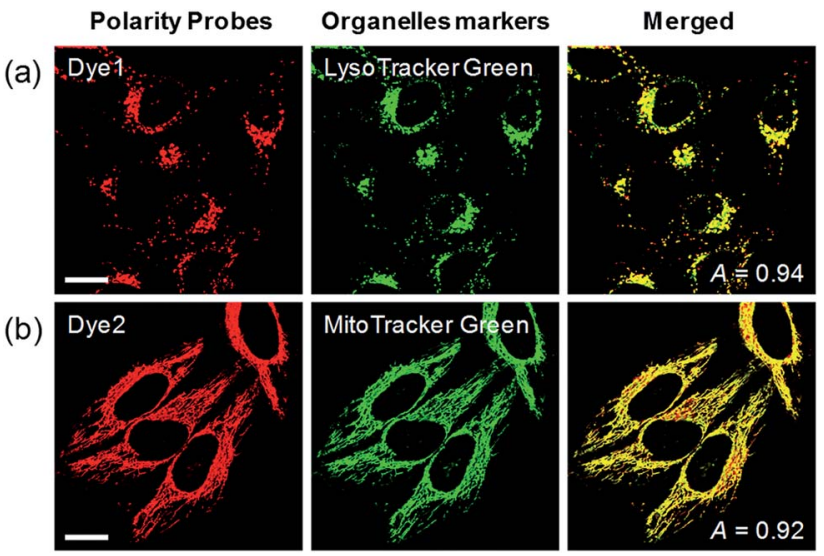

(b)
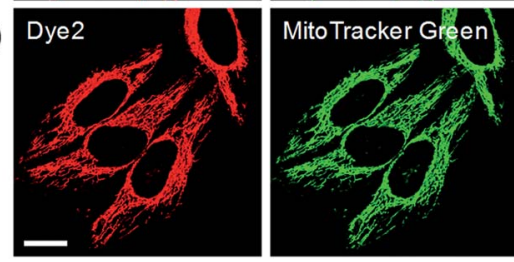

Fig. 3 Co-localization assays in HeLa cells for (a) Dye1 with LysoTracker Green and (b) Dye2 with Mito-Tracker Green, respectively. Excitation wavelengths were $488 \mathrm{~nm}$ (organelle markers) and $552 \mathrm{~nm}$ (polarity probes) and the corresponding emissions were recorded at $500-540 \mathrm{~nm}$ (organelle markers) and 565-650 $\mathrm{nm}$ (polarity probes). Scale bars $=20 \mu \mathrm{m}$.

seen because the lysosomal polarity was specifically higher than other intracellular compartments. The turn-on based probes influenced the fluorescence intensities by various factors such as local concentration, intracellular environment, and imaging conditions. Therefore, to quantitatively confirm the intracellular polarity distribution, a ratiometric probe, whose fluorescence ratio changed according to the environmental polarity, was required.

The benzothiadiazole derivative (Dye3) had an absorption wavelength similar to that of Dye1; however, due to a larger stoke shift, the fluorescence emitted in the long wavelength region of the near infrared (NIR) region minimized the fluorescence interference between the two dyes. In contrast to Dye1, Dye3 was a turn-off probe whose fluorescence intensity decreased as the solvent polarity increased (Fig. S6 $†$ ). Dye1 and Dye3 were combined by introducing the piperazine linker, and the two dye fluorescence intensities responded oppositely according to the environmental polarity. Through this, we synthesized a ratiometric polarity probe (RPS-1), which had two fluorescence spectra with one excitation source (Scheme 1). RPS-1 showed a weak absorbance at $510 \mathrm{~nm}$ in non-polar solvents such as toluene and ether, based on Dye3, and strongly increased the absorbance at $550 \mathrm{~nm}$ in polar solvents such as $\mathrm{MeOH}$ and water, based on Dye1 (Fig. 4a). Moreover, as the polarity increased, the fluorescence intensity near $650 \mathrm{~nm}$ in the nonpolar solvent gradually decreased and the fluorescence around $580 \mathrm{~nm}$ increased (Fig. 4b). The two regions for the ratio fluorescence measurement were defined as $565-585 \mathrm{~nm}\left(F_{\text {yellow }}\right)$ and $630-680 \mathrm{~nm}\left(F_{\text {red }}\right)$, and there was a high correlation between the fluorescence ratio of $F_{\text {yellow }} / F_{\text {red }}$ and the $E_{\mathrm{T}}^{\mathrm{N}}$ value, which is the solvent polarity parameter $\left(R^{2}=0.993\right.$, Fig. $\left.4 \mathrm{c}\right)$. The photophysical properties of RPS-1 are summarized in Table S3. $\dagger$ Additionally, RPS-1 showed reversible changes in the ratio of $F_{\text {yellow }} / F_{\text {red }}$ depending on the solvent polarity, a behavior similar to that of Dye1 (Fig. 4d). The ratio of RPS-1 was almost constant 


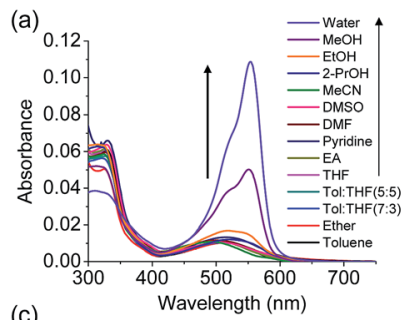

(c)
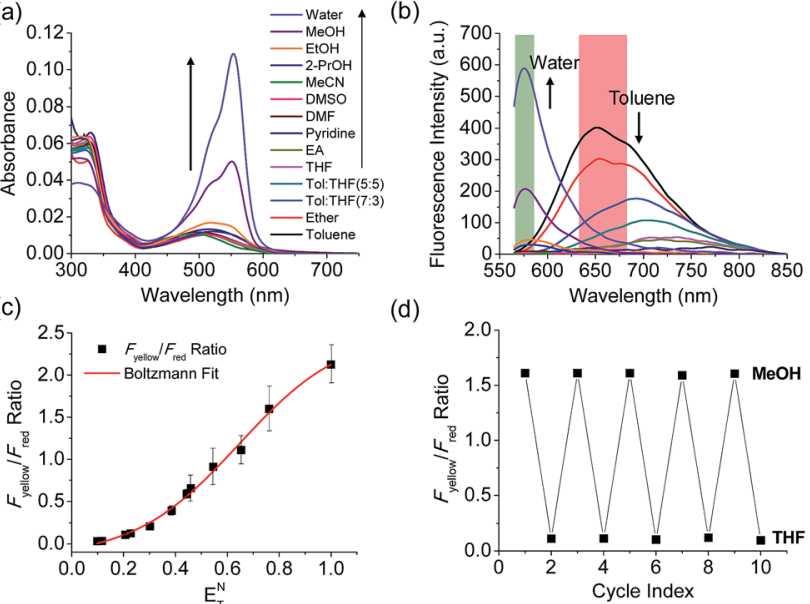

(d)

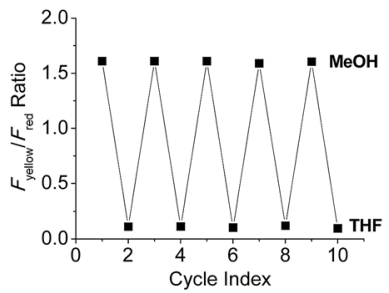

Fig. 4 (a) Absorption spectra and (b) fluorescence spectra of RPS-1 (3 $\mu \mathrm{M})$ in various polar and non-polar solvents. The green box represents the $F_{\text {yellow }}$ region and the red box represents the $F_{\text {red }}$ region. (c) Boltzmann fit of $F_{\text {yellow }} / F_{\text {red }}$ versus the orientation polarizability $E_{\mathrm{T}}^{N}$. (d) Average $F_{\text {yellow }} / F_{\text {red }}$ intensity ratios of RPS-1 with polarity reversibly changed between $\mathrm{MeOH}$ and $\mathrm{THF}$ solvents for 5 cycles. The excitation wavelength was $552 \mathrm{~nm}$.

in various $\mathrm{pH}$ ranges (Fig. $\mathrm{S} 7 \dagger$ ) and in the presence of other biological metabolites, including reactive oxygen and nitrogen species, cationic and anionic amino acids, glutathione, and enzymes (Fig. S8†). Additionally, RPS-1 showed high photostability under the imaging conditions used (Fig. S9†), with negligible cytotoxicity (Fig. S4†).

Ratiometric images were acquired with two channels, $F_{\text {yellow }}$ and $F_{\text {red }}$, to confirm whether RPS-1 reflected the difference in polarity in various compartments within the cell (Fig. 5). The results of HeLa cells labeled with RPS-1 $(3 \mu \mathrm{M})$ for $30 \mathrm{~min}$ surprisingly showed a clear difference between the fluorescence
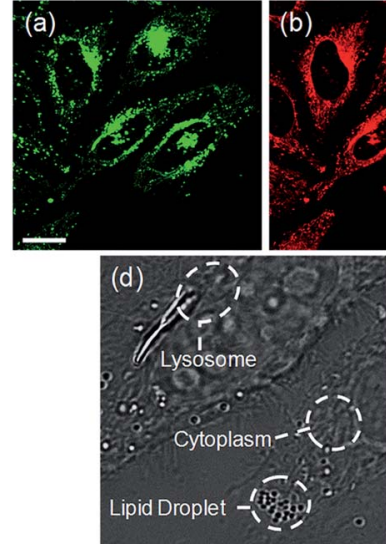
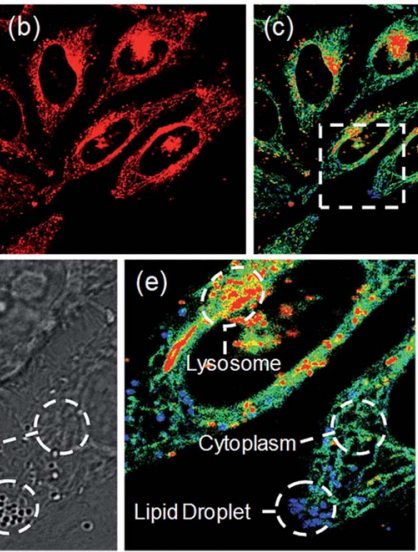

Fig. 5 Fluorescence images and pseudocolored ratiometric images $\left(F_{\text {yellow }} / F_{\text {red }}\right)$ of HeLa cells labeled with RPS-1 $(3 \mu \mathrm{M})$ for $30 \mathrm{~min}$. (a) Fyellow $(565-585 \mathrm{~nm})$ image, (b) $F_{\text {red }}(630-680 \mathrm{~nm})$ image, and (c) ratiometric image. Enlarged (d) bright-field image and (e) ratiometric image of the white box in (c) showing the lysosome, lipid droplet, and cytoplasm. Images were acquired using a $552 \mathrm{~nm}$ excitation source. Scale bars $=20 \mu \mathrm{m}$. of the $F_{\text {yellow }}$ channel and the $F_{\text {red }}$ channel (Fig. 5a and b). Similar to the cell image of Dye1, the $F_{\text {yellow }}$ channel showed a strong fluorescence in a specific vesicle in the cell. However, the fluorescence of the $F_{\text {red }}$ channel was observed in other vesicles, in addition to the same $F_{\text {yellow }}$ vesicle, and stained with strand formation throughout the cell. When the fluorescence images of $F_{\text {yellow }}$ and $F_{\text {red }}$ were treated with a pseudocolored ratiometric image $\left(F_{\text {yellow }} / F_{\text {red }}\right)$, the distribution of the polarities of each region of the cell could be confirmed at a glance (Fig. 5c). All the intracellular polarity images of RPS-1 were confirmed by co-localization experiments with various commercial organelle markers (Fig. 6). In the ratiometric images, the red region with the highest polarity overlapped with the lysosome, while the blue region with the lowest polarity overlapped with the lipid droplet marker. The green region with moderate polarity overlapped with both mitochondria and ER markers, and we defined this region as the cytoplasm. In the enlarged images, the lysosome, cytoplasm, and lipid droplet regions were more clearly distinguished, and the blue-colored lipid droplets in the ratiometric image corresponded exactly to the regions of visually observable lipid droplets in the brightfield image (Fig. 5d and e).
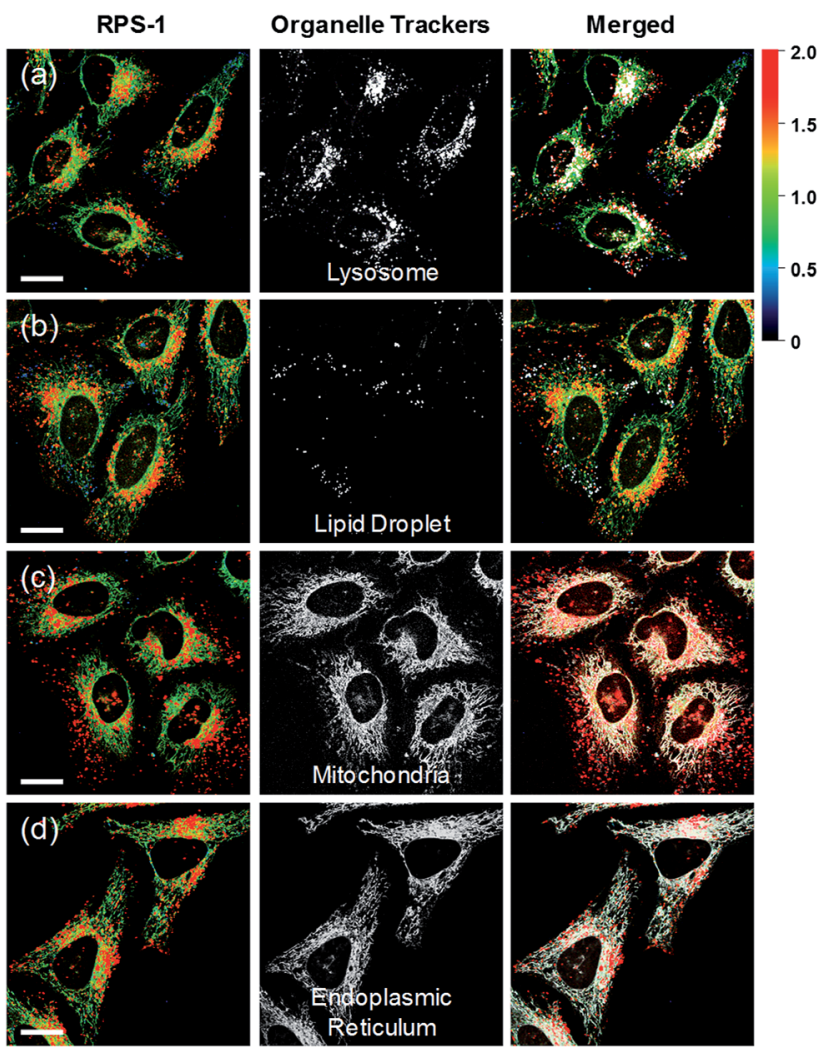

Fig. 6 Co-localization assays of RPS-1 (3 $\mu \mathrm{M})$ and commercial organelle trackers (1 $\mu \mathrm{M})$ for (a) lysosomes, (b) lipid droplets, (c) mitochondria, and (d) the endoplasmic reticulum in HeLa cells. Excitation wavelengths were $488 \mathrm{~nm}$ (organelle trackers) and $552 \mathrm{~nm}$ (RPS-1) and the corresponding emissions were recorded at 500$540 \mathrm{~nm}$ (organelle trackers), 565-585 nm (RPS-1, yellow), and 630$680 \mathrm{~nm}$ (RPS-1, red). Scale bars $=20 \mu \mathrm{m}$. 

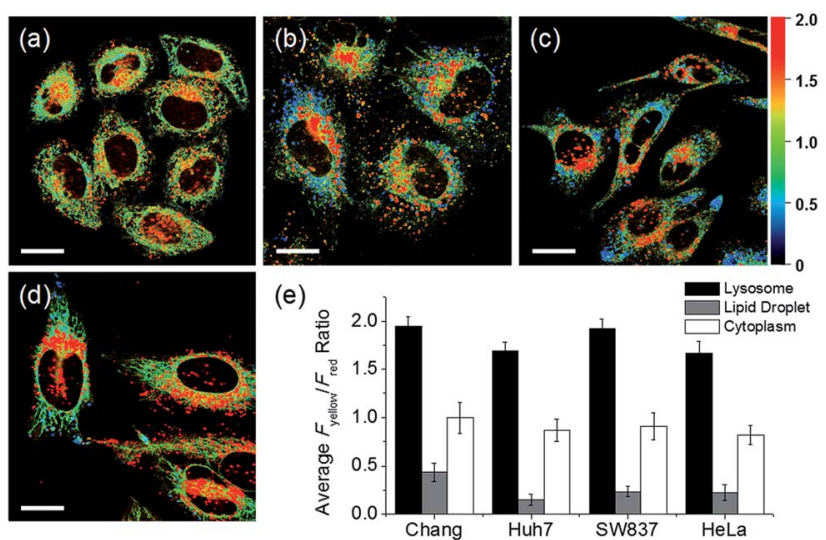

Fig. 7 Pseudocolored ratiometric images $\left(F_{\text {yellow }} / F_{\text {red }}\right)$ of (a) Chang, (b) Huh7, (c) SW837, and (d) HeLa cells labeled with RPS-1 $(3 \mu \mathrm{M})$ for $30 \mathrm{~min}$. (e) Average $F_{\text {yellow }} / F_{\text {red }}$ intensity ratios of each corresponding image. Images were acquired using $552 \mathrm{~nm}$ excitation and emission windows of $565-585 \mathrm{~nm}$ (yellow) and $630-680 \mathrm{~nm}$ (red). Scale bars = $20 \mu \mathrm{m}$.

In addition to HeLa cells, the distribution of intracellular polarity in various cells such as Chang, Huh7, and SW837 was observed; the polarity of the lysosomes was observed to be the highest in all cells (Fig. 7). The $F_{\text {yellow }} / F_{\text {red }}$ fluorescence ratios of lysosomes in the Chang, Huh7, SW837, and HeLa cells were 1.9, $1.7,1.9$, and 1.7 , respectively. The polarity of lysosomes in all cells was as high as the value between those of methanol and water (Fig. 4c). Conversely, the lipid droplets had lower polarity compared to other organelles. The $F_{\text {yellow }} / F_{\text {red }}$ fluorescence ratio of the lipid droplets in Huh7, SW837, and HeLa cells was 0.15, 0.24 , and 0.22 , respectively, while that of Chang cells was 0.43 , which was higher than that of the other cells. This is consistent with previous studies that showed that the polarity of lipid droplets of cancer cells is lower than that of normal cells due to the specific lipid metabolism of cancer cells. ${ }^{21,32}$ The cytoplasmic polarity value was between those of the lysosomes and lipid droplets, and the $F_{\text {yellow }} / F_{\text {red }}$ fluorescence ratio values in the cytoplasm of Chang, Huh7, SW837, and HeLa were 1.0, 0.87, 0.91 , and 0.81 , similar to that of $2-\mathrm{PrOH}$. In particular, the $F_{\text {yellow }} / F_{\text {red }}$ fluorescence ratios provided information about the polarity of solvents similar to that of lysosomes (1.7-1.9, $\mathrm{MeOH}$ and water), cytoplasm (0.81-1.0, 2-PrOH), and lipid droplets (0.15-0.43, DMF and EA). Consequently, intracellular polarity was heterogeneous and the polarity of each organelle gradually decreased from lysosomes, to cytoplasm, to lipid droplets.

\section{Conclusions}

We developed Dye1, in which the absorbance and fluorescence intensity changed through intramolecular cyclization-opening according to solvent polarity. Dye1 is the first hydrophilic environmentally selective turn-on probe in the spiropyran series, in which the structure of the molecule itself changes reversibly in response to polarity. We synthesized a hydrophobic and hydrophilic combined ratiometric probe, RPS-1, in which the intensity of the two fluorophores was reversely changed by polarity by introducing Dye3, whose fluorescence decreased as the solvent polarity increased. By connecting the two dyes with similar absorption but different solvatochromic shifts, RPS-1 was able to measure the difference in fluorescence ratios $\left(F_{\text {yellow }} / F_{\text {red }}\right)$ according to polarity using one excitation wavelength and quantitatively detect the change in polarity over a wide range, from toluene to water. RPS-1 was stained in various organelles in the cell and showed a difference in polarities in various regions of the cell at a glance. These results suggested that RPS-1 could detect a wide range of intracellular polarity changes sensitively and quantitatively, and confirmed that the polarity of the lysosomes was the highest in the cell. This new approach of linking two dyes with completely different characteristics resulted in a new ratiometric polarity sensing dye, RPS-1, that could provide useful information to biomedical research.

\section{Experimental sections}

Spectroscopic measurements. Absorption spectra and fluorescence spectra were recorded with a UV-Vis spectrophotometer (S-3100) and fluorescence spectrophotometer (FS-2), respectively. The fluorescence quantum yield was measured with 9,10-diphenylanthracene ( $\Phi=0.93$ in cyclohexane) as the reference. The ${ }^{1} \mathrm{H}$ NMR spectra were recorded using $600 \mathrm{MHz}$ NMR spectrometers (JNM-ECZR). The fluorescence images were obtained with spectral confocal microscopes (Leica TCS SP8).

Cell images. Each cell was incubated for two days before imaging. Streptomycin $\left(100 \mu \mathrm{g} \mathrm{mL}^{-1}\right)$, penicillin (100 units per $\mathrm{mL}$ ), and $10 \%$ fetal bovine serum were added to all culture media. The culture medium was replaced with a serum-free medium and each polarity probe was stained for $30 \mathrm{~min}$. Live cell imaging was performed using a live-cell instrument (Chamlide IC) to maintain appropriate temperature, humidity, and $\mathrm{pH}$ for long term exposure. Ratiometric image processing and analysis were carried out using MetaMorph software.

Co-localization experiments. Experiments were carried out by co-staining with polarity probes and each commercial organelle tracker (LysoTracker Green DND-26 for lysosomes, BODIPY 493/503 for lipid droplets, MitoTracker Green FM for mitochondria and ER-Tracker Green for the endoplasmic reticulum). The excitation wavelengths were $488 \mathrm{~nm}$ (organelle trackers) and $552 \mathrm{~nm}$ (polarity probes). Pearson's colocalization coefficient (A) was calculated using AutoQuant X2 software.

\section{Conflicts of interest}

There are no conflicts to declare.

\section{Acknowledgements}

This study was supported by grants from the National Leading Research Lab Program of the National Research Foundation of Korea (NRF), funded by the Korean government (MSIP) (NRF2019R1A2B5B03100278), the Center for Convergence Research of Neurological Disorders (NRF-2019R1A5A2026045), and the Ajou University Research Fund. 


\section{Notes and references}

1 K. Luby-Phelps, Int. Rev. Cytol., 2000, 192, 189-221.

2 Z. Yang, J. Cao, Y. He, J. H. Yang, T. Kim, X. Peng and J. S. Kim, Chem. Soc. Rev., 2014, 43, 4563-4601.

3 D. G. Drubin and W. J. Nelson, Cell, 1996, 84, 335-344.

4 M. Simons and M. Mlodzik, Annu. Rev. Genet., 2008, 42, 517540.

5 M. Bornens, Nat. Rev. Mol. Cell Biol., 2008, 9, 874-886.

6 H. J. Sharpe, T. J. Stevens and S. A. Munro, Cell, 2010, 142, 158-169.

7 C. Cottet-Rousselle, X. Ronot, X. Leverve and J. F. Mayol, Cytometry, Part A, 2011, 79, 405-425.

8 H. Xiao, C. Wu, P. Li, W. Gao, W. Zhang, W. Zhang, L. Tong and B. Tang, Chem. Sci., 2017, 8, 7025-7030.

9 M. Dykstra, A. Cherukuri, H. W. Sohn, S. J. Tzeng and S. K. Pierce, Annu. Rev. Immunol., 2003, 21, 457-481.

10 M. T. Butler and J. B. Wallingford, Nat. Rev. Mol. Cell Biol., 2017, 18, 375-388.

11 M. Lee and V. Vasioukhin, J. Cell Sci., 2008, 121, 1141-1150.

12 N. Jiang, J. Fan, F. Xu, X. Peng, H. Mu, J. Wang and X. Xiong, Angew. Chem., Int. Ed., 2015, 54, 2510-2514.

13 H. Xiao, P. Li, W. Zhang and B. Tang, Chem. Sci., 2016, 7, 1588-1593.

14 H. Zhu, J. Fan, H. Mu, T. Zhu, Z. Zhang, J. Du and X. Peng, Sci. Rep., 2016, 6, 1-10.

15 J. Jiang, X. Tian, C. Xu, S. Wang, Y. Feng, M. Chen, H. Yu, M. Zhu and X. Meng, Chem. Commun., 2017, 53, 3645-3648.

16 M. Li, J. Fan, H. Li, J. Du, S. Long and X. Peng, Biomaterials, 2018, 164, 98-105.

17 L. Fan, X. Wang, J. Ge, F. Li, X. Wang, J. Wang, S. Shuang and C. Dong, Chem. Commun., 2019, 55, 4703-4706.

18 Z. Yang, Y. He, J. H. Lee, W. S. Chae, W. X. Ren, J. H. Lee, C. Kang and J. S. Kim, Chem. Commun., 2014, 50, 1167211675.
19 W. Song, B. Dong, Y. Lu, X. Kong, A. H. Mehmood and W. Lin, New J. Chem., 2019, 43, 12103-12108.

20 K. Pal, I. Samanta, R. K. Gupta, D. Goswami and A. L. Koner, Chem. Commun., 2018, 54, 10590-10593.

21 J. Yin, M. Peng, Y. Ma, R. Guo and W. Lin, Chem. Commun., 2018, 54, 12093-12096.

22 M. Collot, S. Bou, T. K. Fam, L. Richert, Y. Mely, L. Danglot and A. S. Klymchenko, Anal. Chem., 2019, 91, 1928-1935.

23 M. Collot, T. K. Fam, P. Ashokkumar, O. Faklaris, T. Galli, L. Danglot and A. S. Klymchenko, J. Am. Chem. Soc., 2018, 140, 5401-5411.

24 H. M. Kim, B. H. Jeong, J. Y. Hyon, M. J. An, M. S. Seo, J. H. Hong, K. J. Lee, C. H. Kim, T. Joo, S. C. Hong and B. R. Cho, J. Am. Chem. Soc., 2008, 130, 4246-4247.

25 O. A. Kucherak, S. Oncul, Z. Darwich, D. A. Yushchenko, Y. Arntz, P. Didier, Y. Mely and A. S. Klymchenko, J. Am. Chem. Soc., 2010, 132, 4907-4916.

26 A. S. Klymchenko and R. Kreder, Chem. Biol., 2014, 21, 97113.

27 X. Zheng, W. Zhu, F. Ni, H. Ai, S. Gong, X. Zhou, J. L. Sessler and C. Yang, Chem. Sci., 2019, 10, 2342-2348.

28 W. A. Prinz, J. Cell Biol., 2014, 205, 759-769.

29 M. Schrader, L. F. Godinho, J. L. Costello and M. Islinger, Front. Cell Dev. Biol., 2015, 3, 56.

30 L. D. Zorova, V. A. Popkov, E. Y. Plotnikov, D. N. Silachev, I. B. Pevzner, S. S. Jankauskas, V. A. Babenko, S. D. Zorov, A. V. Balakireva, M. Juhaszova, S. J. Sollott and D. B. Zorov, Anal. Biochem., 2018, 552, 50-59.

31 W. Chen, C. Gao, X. Liu, F. Liu, F. Wang, L. J. Tang and J. H. Jiang, Anal. Chem., 2018, 90, 8736-8741.

32 L. Tirinato, F. Pagliari, T. Limongi, M. Marini, A. Falqui, J. Seco, P. Candeloro, C. Liberale and E. Di Fabrizio, Stem Cells Int., 2017, 2017, 1656053. 